$\underline{\text { RELATO DE CASO }}$

\title{
Aneurisma de artéria pulmonar como manifestação da doença de Behçet*
}

\author{
Marcos Naoyuki Samano ${ }^{1}$, Renata Teixeira Ladeira ${ }^{2}$, Luiz Pedro Meireles ${ }^{3}$, Paulo Manuel PÊgo-Fernandes ${ }^{4}$
}

A doença de Behçet pode ser acompanhada de manifestações pulmonares, às vezes letais se não tratadas adequadamente. Descreve-se o caso de um homem de 53 anos com história de episódios de aftas orais e febre havia vários anos, dois quadros febris prolongados nos últimos dois anos, uveíte tratada com corticóide havia 21 meses, hemoptise havia um mês e emagrecimento de $7 \mathrm{~kg}$. Ao exame físico apresentava aftas orais, pseudofoliculite e turvação visual. Os estudos radiológicos evidenciaram dilatação aneurismática de artéria pulmonar. Tratado com corticóide, foi feita lobectomia inferior esquerda e o exame anatomopatológico foi compatível com arterite de artéria pulmonar. 0 paciente

foi tratado com clorambucil por oito meses e mantém-se bem, decorridos 18 meses da cirurgia.

(J Pneumol 2002;28(3):150-154)

\section{Pulmonary artery aneurysm as a manifestation of Behçet disease: case report and review}

Behçet disease has been associated to pulmonary manifestations and may be lethal if not treated correctly. The authors describe a case of a 53 year-old male with aneurysmatic pulmonary artery dilatation, pulmonary hypertension, and hemoptysis. A review is presented about the diagnosis and the medical and surgical treatment of this disease.

Descritores - Síndrome de Behçet. Artéria pulmonar. Hipertensão pulmonar. Aneurisma. Hemoptise.

Key words - Behcet's syndrome. Pulmonary artery. Pulmonary hypertension. Aneurysm. Hemoptysis.

\section{INTRODUÇÃO}

A doença de Behçet (DB) é uma vasculite multissistêmica de origem desconhecida que afeta as artérias e veias de diversos calibres ${ }^{(1)}$. Hipócrates, no ano 5 a.C., descreveu uma síndrome compreendendo ulcerações orais e genitais associada a inflamação ocular, também relatada

\footnotetext{
* Trabalho realizado no Hospital das Clínicas da Faculdade de Medicina da Universidade de São Paulo.

1. Médico Residente do Serviço de Cirurgia Torácica.

2. Médica Residente da Disciplina de Clínica Geral.

3. Médico Assistente do Serviço de Clínica Geral.

4. Professor Livre-Docente da Disciplina de Cirurgia Cardiotorácica.

Endereço para correspondência - Av. Dr. Enéas Carvalho de Aguiar, 44, Incor-HCFMUSP, 2ำ andar, divisão cirúrgica - 04503-000 - São Paulo, SP. Tel. (11) 3069-5248. E-mail: paulopego@incor.usp.br Recebido para publicação em 9/1/01. Aprovado, após revisão, em 18/3/02.
}

Abreviatura utilizada neste trabalho

DB - Doença de Behçet

pelo oftalmologista grego Adamantiades(2) em 1931. Porém, é ao dermatologista turco Hulusi B ehçet que se atribui a caracterização da síndrome em 1937, compreendendo a coexistência de estomatites, ulcerações genitais e envolvimento ocular ${ }^{(3)}$.

Sua etiologia está relacionada a mecanismos auto-imunes, pós-infecção e fatores genéticos. Sugere-se também a influência de fatores ambientais. A presença de tendência trombótica é malcompreendida, citando-se mudanças no fator XII, nível da proteína S e C, defeito na fibrinólise e falta de sensibilidade das plaquetas à prostaciclina(2).

Os critérios para diagnóstico da $D^{(4)}$ incluem a presença de ulcerações orais recorrentes, do tipo aftosa ou herpetiforme, que ocorrem pelo menos três vezes no período de 12 meses, associadas a pelo menos duas das seguintes alterações, na ausência de doença sistêmica: ulcerações genitais recorrentes, lesões oculares (uveíte anterior ou posterior, vasculite na retina), lesões cutâneas (eritema nodoso, pseudofoliculite, lesões pápulo-pustulo- 


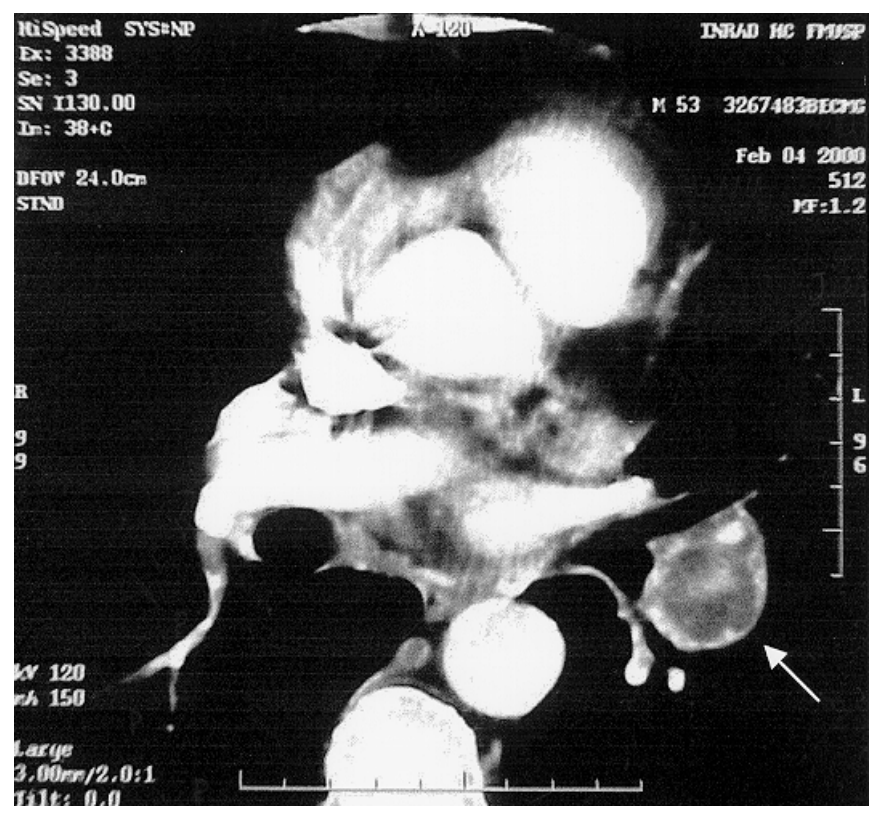

Figura 1 - Tomografia computadorizada de tórax evidenciando dilatação aneurismática de artéria descendente interlobar esquerda com trombo intramural (seta)

sas e lesões semelhantes a acnes em não-adolescentes) ou positividade no teste de patergia. Neste teste, após limpeza da pele do antebraço são realizadas duas picadas, uma com agulha afiada e outra com agulha sem corte. A leitura é feita em 48 horas e é positivo quando há formação de pápula estéril maior do que $2 \mathrm{~mm}$. Histologicamente, observa-se infiltrado de monócitos em torno dos $\operatorname{vasos}^{(5)}$.

A doença de Behçet tem distribuição universal, embora a maioria dos casos relatados seja no J apão, no Oriente Médio e no Mediterrâneo. A prevalência da doença tem aumentado durante os últimos 40 anos, principalmente no J apão, onde a incidência chega a 10/100.000, dependendo da região. Comparativamente, no Irã a prevalência varia de 16 a 100/100.000, na Turquia é de 5/ 100.000, enquanto no Canadá, de 0,3/100.000.

Neste trabalho, relata-se a experiência com o diagnóstico e tratamento clínico-cirúrgico de um paciente com DB com acometimento aneurismático e trombótico da artéria pulmonar descendente esquerda.

\section{RELATO dO CASO}

Homem de 53 anos apresentou, dois anos antes da internação, quadro súbito de tontura, associado a febre alta e calafrios que se estenderam por dois meses. Nesse momento passou a apresentar tosse com expectoração clara, realizando radiografia e tomografia de tórax, que evidenciaram condensação em lobo inferior esquerdo,

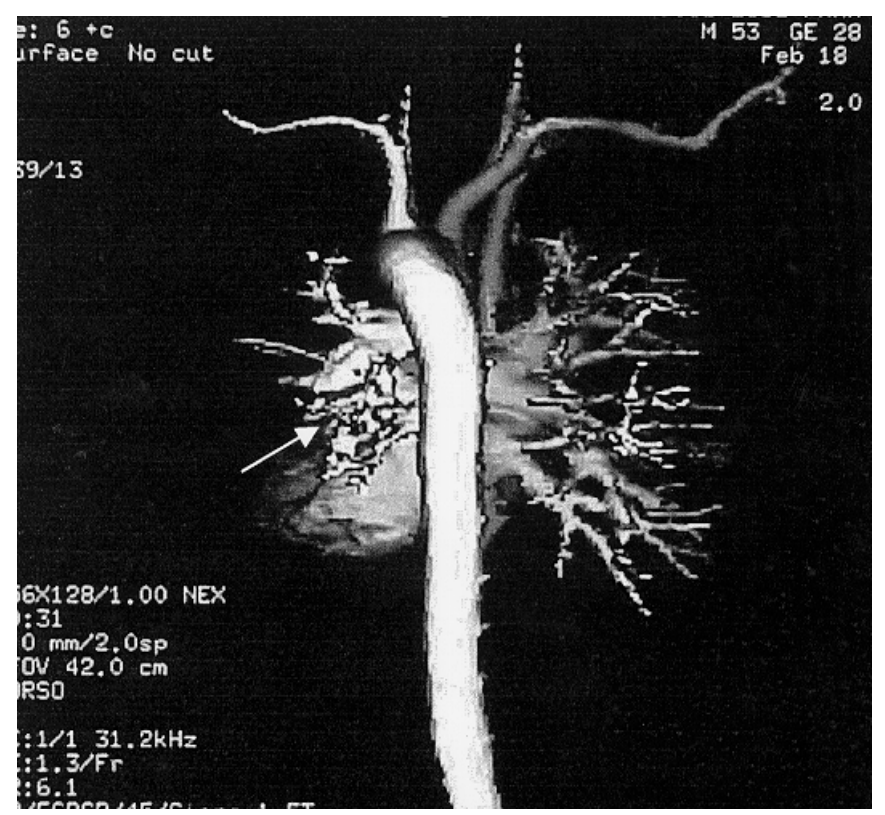

Figura 2 - Vista posterior de angiorressonância evidenciando diminuição da circulação arterial do pulmão esquerdo, além de ausência de circulação para o lobo inferior (seta)

sendo tratado como pneumonia, com melhora clínica e radiológica. Após três meses apresentou uveíte posterior bilateral, sendo tratado com corticóide com sucesso. Após seis meses, novamente com quadro febril prolongado, foi realizada ampla investigação clínica, sem sucesso no diagnóstico. Houve remissão espontânea, permanecendo assintomático até que 30 dias antes da internação apresentou subitamente hemoptise, com volume aproximado de $300 \mathrm{ml}$, seguida de dispnéia leve, febre e emagrecimento de $7 \mathrm{~kg}$, sendo novamente internado para investigação.

Como antecedentes, tabagismo por 25 anos, relatava aftas orais associadas a febre, pseudofoliculite em dorso e membros superiores recorrentes havia vários anos. Referia ter apresentado flebite em membro inferior esquerdo havia 10 anos. Ao exame físico não apresentava alterações, exceto aftas orais difusas, pseudofoliculite e turvação visual.

Os exames laboratoriais eram normais, exceto pela elevação da velocidade de hemossedimentação e pela presença de anticoagulante lúpico positivo. Radiografia simples de tórax mostrou alargamento do hilo à esquerda. A broncoscopia não evidenciava sangramento ativo, sugerindo apenas bronquite crônica. Tomografia helicoidal de tórax evidenciou dilatação aneurismática da artéria descendente interlobar esquerda próximo à sua emergência no tronco pulmonar com trombo intramural (Figura 1). A cintilografia pulmonar de perfusão evidenciou hipoperfusão difusa do pulmão esquerdo e alterações discretas no pulmão direito. Ecocardiograma mostrava hipertensão 


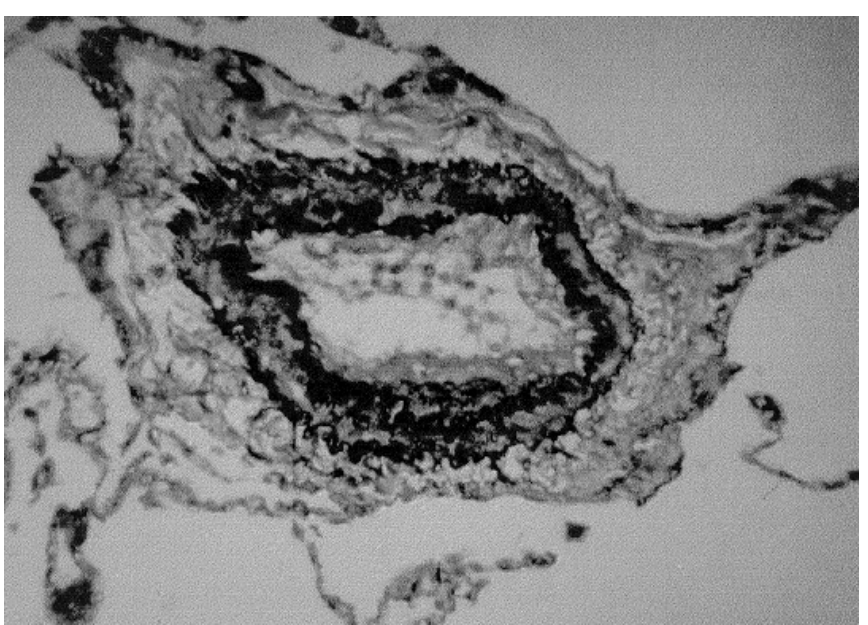

Figura 3 - Achados histopatológicos caracterizados pela rotura da lâmina elástica interna e externa, afilamento da íntima e degeneração da camada média

pulmonar leve $(40,5 \mathrm{mmHg})$. Foi realizada angiorressonância, que não evidenciou outros aneurismas (Figura 2).

Foi iniciada corticoterapia com prednisona na dose de $1 \mathrm{mg} / \mathrm{kg}$ de peso e, após 30 dias de tratamento, indicada a lobectomia inferior esquerda. Os achados intra-operatórios evidenciavam uma dilatação importante da artéria próxima à sua emergência do tronco, porém sem acometimento do mesmo. A consistência da parede da artéria era normal, possibilitando que a ressecção fosse feita com segurança, embora todos os cuidados com o controle proximal houvessem sido tomados. Os achados anatomopatológicos mostravam arterite linfocitária, destruição de fibras elásticas, neovascularização e fibrose das túnicas íntima e média e adventícia, infartos pulmonares cicatrizados, compatíveis com comprometimento da artéria pulmonar na DB (Figura 3). No pós-operatório foi mantida prednisona na dose inicial e, no quarto mês após a lobectomia, iniciado clorambucil na dose de $4 \mathrm{mg} / \mathrm{dia}$. A escoIha do clorambucil foi feita em função da piora da uveíte em vigência de corticoterapia. 0 clorambucil é a droga imunossupressora de escolha no acometimento oftalmológico da doença de Behçet. Não há consenso quanto à droga de escolha para a vasculite. Realizou-se, então, a retirada progressiva do corticóide, mantendo-se o clorambucil por mais oito meses. Decorridos 18 meses da cirurgia, o paciente encontra-se bem.

\section{DISCUSSÃO}

O comprometimento vascular na DB consiste em tromboflebites, aneurismas e oclusões arteriais, vistos em aproximadamente $25 \%$ dos pacientes $(3,6,7)$. 0 envolvimento pulmonar é raro e ocorre em torno de $5-10 \%$ dos pacientes com $\mathrm{DB}^{(8)}$, sendo que os aneurismas têm prevalên-

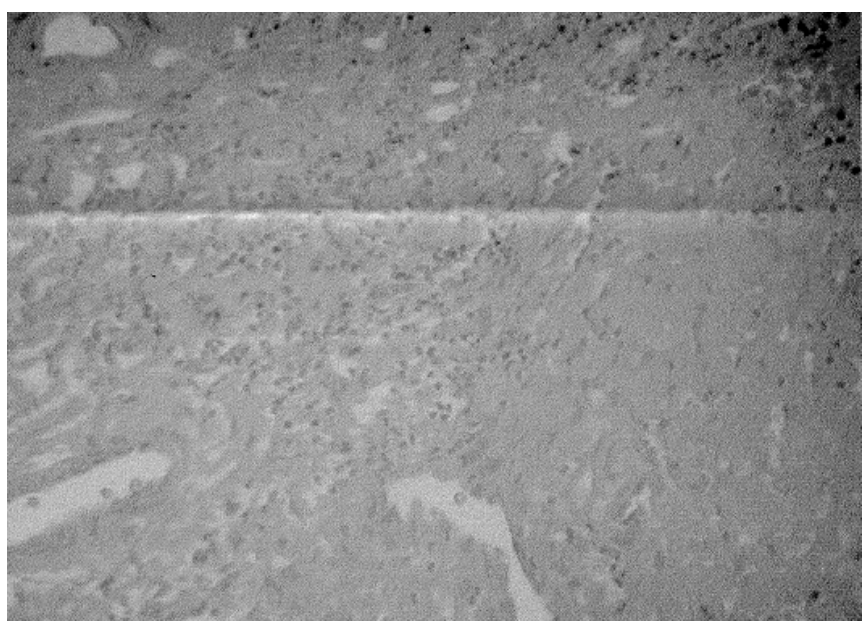

Figura 4 - Achados histopatológicos compatíveis com vasculite do vasa vasorum e infiltrado perivascular linfocítico

cia de $1,5 \%^{(3,9,10)}$. Os aneurismas acometem principalmente 0 pulmão direito (59\%), sendo mais comuns em artérias lobares (54\%), com a presença de trombose em um terço dos pacientes ${ }^{(11,12)}$.

A etiopatogenia da formação dos aneurismas de artéria pulmonar é incerta ${ }^{(13)}$. Várias alterações imunológicas têm sido descritas na DB, como a ação de enzimas lisossômicas e radicais livres, auto-anticorpos contra as células do endotélio e vasculite necrotizante com infiltrado linfocítico, principalmente na média e adventícia dos va$\operatorname{sos}^{(13)}$. H istologicamente, a formação de aneurismas é caracterizada pela rotura da lâmina elástica interna e externa, com afilamento da íntima, degeneração da média e vasculite do vasa vasorum com infiltrado perivascular linfocítico(3,14-16) (Figuras 3 e 4).

Os aneurismas de artéria pulmonar podem complicarse com a formação de trombos, levando a infartos pulmonares, e com rotura para o interior dos brônquios, causando hemoptises maciças para o espaço pleural, com a formação de hemotórax, ou para o interior do parênquima, com hemorragias pulmonares $(2,13)$.

As manifestações clínicas pulmonares são dispnéia, tosse, dor torácica e hemoptise ${ }^{(17)}$, sendo este último 0 sintoma mais freqüente, tendo importância prognóstica quando causado pelos aneurismas de artéria pulmonar. Há descrição de $50 \%$ de mortalidade, num período de 9,5 meses em média após o primeiro episódio de hemoptise.

Em geral, ocorre aumento da velocidade de hemossedimentação, podendo haver hipergamaglobulinemia, leucocitose, anemia, trombocitopenia e, raramente, leucopenia. A anticardiolipina, principalmente a IgM, está presente em $30 \%$ dos casos de DB, sendo relacionada a maior tendência a eventos embólicos ${ }^{(2)}$. 
O achado mais freqüente na radiografia de tórax é a opacidade peri-hilar uni ou bilateral (usualmente, representa aneurismas de artérias pulmonares) $)^{(3,18,19)}$. A tomografia convencional de tórax é bastante útil no diagnóstico e no seguimento dos aneurismas, por ser menos invasiva e mostrar a presença de trombos parietais. Entretanto, é sensível apenas na identificação de aneurismas de artérias lobares e segmentares, enquanto a tomografia helicoidal é mais apropriada por detectar tanto aneurismas de pequeno calibre como aqueles trombosados ${ }^{(11,17)}$.

Apesar de ser o padrão-ouro na detecção de aneurismas de artéria pulmonar, a angiografia envolve riscos na DB. A punção venosa ou injeção rápida de grande quantidade de contraste pode iniciar a formação de trombo ou agravar um que já exista, além de não mostrar aneurismas que estejam trombosados ${ }^{(11)}$. Pseudo-aneurismas podem desenvolver-se no sítio de punção arterial(2,3,14).

A angiorressonância é um exame seguro, não invasivo, que pode ser usado para o diagnóstico e monitorização dos aneurismas de artéria pulmonar, especialmente para pacientes com história de alergia a contraste, oferecendo vantagem em relação à angiografia convencional por não necessitar da cateterização arterial ou venosa e da injeção de contraste ${ }^{(18)}$. A capacidade de fornecer imagens multiplanares e de diagnosticar aneurismas independente da sua patência tem mostrado a superioridade deste exame nos pacientes portadores de $\mathrm{DB}^{(9)}$.

Várias modalidades de tratamento, com diferentes resultados, têm sido descritas. Estas incluem uso de imunossupressores isolados, ou em associação com corticóide, cirurgia e embolização, porém, não há trabalhos controlados que indiquem a melhor opção ${ }^{(20,22)}$.

A terapia com corticóide, primária ou no pós-operatório, claramente tem papel em prevenir a recorrência de lesões arteriais ${ }^{(23)}$. Porém, pelo risco de rotura, os aneurismas sempre devem receber abordagem cirúrgica, se únicos e sintomáticos. É apropriado iniciar corticoterapia com prednisona da dose de $1 \mathrm{mg} / \mathrm{kg}$ nos casos de aneurismas recém-diagnosticados. Em alguns casos, pode haver resolução dos sinais e sintomas, bloqueando a progressão da lesão e podendo ocorrer até mesmo regressão dos aneurismas com reconstituição da vasculatura pulmonar na íntegra ${ }^{(10,17,24)}$. Entretanto, as recidivas podem ocorrer em até $50 \%$ dos casos tratados isoladamente com corticóide e a adição de imunossupressores meIhora esta incidência(23). Tunaci et al. relataram regressão total em $76 \%$ dos casos e parcial em $24 \%$ após o uso de ciclosporina e prednisona em 46 aneurismas de 13 pacientes, com cirurgia apenas em dois casos devido a hemoptise maciça ${ }^{(11)}$. Em nosso meio, Barros et al. relataram, em 1997, um caso de paciente portador de DB que apresentava dilatação aneurismática do tronco pulmonar, tratado com prednisona e ciclofosfamida, com melhora clínica e radiológica(29). Magalhães et al., em 1999, também relataram a regressão de aneurismas pulmonares através do uso de imunossupressores ${ }^{(30)}$. Há relato do uso de ciclofosfamida, ciclosporina, azatioprina, clorambucil, sendo a primeira a mais usada, porém, sem haver nenhum trabalho controlado, multicêntrico, que defina qual a melhor droga ${ }^{(3,16,24,25)}$.

Embora haja tendência trombótica nesses pacientes, 0 risco de novo sangramento contra-indica a utilização de anticoagulantes ${ }^{(2,19)} \mathrm{H}$ amuryudan et al. ${ }^{(7)}$ destacam que, entre 24 pacientes de sua série, cinco tiveram inicialmente diagnóstico de tromboembolismo pulmonar recebendo anticoagulante. Destes, dois morreram com hemoptise maciça, destacando a importância de evitar o uso dessa medicação.

Apesar de não haver consenso no tratamento cirúrgico dos portadores de aneurisma de artéria pulmonar ${ }^{(26)}$, a ruptura e evolução fatal dos aneurismas justificam a terapêutica invasiva nesses pacientes ${ }^{(27,28)}$. Segundo H amuryudan et al., mais de $50 \%$ dos pacientes que apresentaram hemoptise tiveram óbito em três anos. Rás et al., em série analisada de 49 pacientes, relataram óbito aos dois anos de $30 \%$ dos que apresentaram hemoptise. Em nosso meio, Lorenzi Filho et al. relataram a evolução quase sempre fatal desses pacientes que apresentam aneurisma pulmonar(31). O tratamento cirúrgico depende da exata localização desses aneurismas. Quando localizados no tronco da artéria pulmonar, a maioria dos grupos opta pela aneurismorrafia, embora haja relatos do uso de próteses de dácron e patch de pericárdio. Quando os aneurismas estão localizados distalmente ao tronco da pulmonar, a ressecção torna-se a terapêutica de escolha. Nos casos de aneurisma de artéria principal, alguns cirurgiões têm optado pela pneumectomia. Nos aneurismas periféricos, a opção é pela lobectomia. A presença de múltiplos aneurismas não é rara e, nestes casos, não há indicação para múltiplas ressecções. Nesses casos, a arteriografia com embolização tem mostrado bons resultados, sendo considerada boa alternativa nos aneurismas que não envolvem o tronco ou os ramos principais da artéria pulmonar ${ }^{(28)}$.

\section{Agradecimentos}

Os autores agradecem a Aurelino Fernandes Schmidt J r. (médico ex-residente do Serviço de Cirurgia Torácica) e Érica O kazaki (acadêmica do 60 ano da Faculdade de Medicina da Universidade de São Paulo) pela colaboração no acompanhamento do paciente e levantamento bibliográfico.

\section{REFERÊNCIAS}

1. Tüzün H, Hamuryudan V, Yildirin S, Besirli K, Yörük Y, Yurdakul S, et al. Surgical therapy of pulmonary arterial aneurysms in Behçet's syndrome. Ann Thorac Surg 1996;61:733-5. 
2. Bradbury AW, Milne AA, Murie JA. Surgical aspects of Behçet's disease. $\mathrm{Br} J$ Surg 1994;81:1712-21.

3. Numan $F$, Islak $C$, Berkmen $T$, Tüzün $H$, Cokyüksel $O$. Behçet disease: pulmonary arterial involvement in 15 cases. Radiology 1994;192:4658.

4. International Study Group for Behçet's Disease. Criteria for diagnosis of Behçet's disease. Lancet 1990;335:1078-80.

5. Gul A, Esin S, Dilsen N, Konice NT, Wigzell K, Diberfeld B. Immunohistology of skin pathergy reaction in Behçet disease. $\mathrm{Br} J$ Dermatol 1995;132:901-7.

6. Shimizu T, Rlich GE, Inaba G, Hayashi K. Behçet's disease. Semin Arthritis Rheum 1979;8:223-31.

7. Hamuryudan V, Yurdakul S, Moral F, Numan F, Tüzün $H$, Tüzüner $N$, et al. Pulmonary arterial aneurysms in Behçet syndrome: a report of 24 cases. Br J Reumatol 1994;33:48-51.

8. Montprèville VT, Macchiarini P, Dartevelle PG, Dulmet EM. Large bilateral pulmonary artery aneurysms in Behçet's disease: rupture of the contralateral lesion after aneurysmorraphy. Respiration 1996;63:4951.

9. Berkmen T. MR angiography of aneurysms in Behçet disease: a report of four cases. J Comput Assist Tomogr 1998;22:202-6.

10. Stricker $H$, Malinverni R. Multiple, large aneurysms of pulmonary arteries in Behçet's disease. Arch Intern Med 1989;149:925-7.

11. Tunaci M, Özkorkmaz B, Tunaci A, Gül A, Engin G, Acunas B. CT findings of pulmonary artery aneurysms during treatment for Behçet's disease. AJR 1999;172:729-33.

12. Lakhkar BN, Nagaraj MV, Shenoy DP, Patil UD, Ghosh MK. Bilateral pulmonary aneurysm in Behçet's disease. J Postgrad Med 1992;38: 47-8.

13. Slavin RE, deGroot WJ. Pathology of the lung in Behçet disease: case report and review of literature. Am J Surg Pathol 1981;5:779-88.

14. Tunaci A, Berkmen T, Gokmen E. Thoracic involvement in Behçet's disease: pathologic, clinical and imaging features. AJR 1995;164:516.

15. Matsumoto T, Vekusa T, Fukuda Y. Vasculobehçet's disease: a pathologic study of eight cases. Hum Pathol 1991;1:45-51.

16. Hamza M. Large artery involvement in Behçet's disease. J Rheumatol 1987; 14:554-9.

17. Almog Y, Polliack G, Dranitzki EM, Shalit M, Rosenmann E. Bilateral pulmonary artery aneurysms in Behçet's disease. Eur Respir 1993;6: 1067-9.
18. Puckette TC, Jolles H, Proto AV. Magnetic resonance imaging confirmation of pulmonary artery aneurism in Behçet's disease. J Thorac Imaging 1994;9:172-5.

19. Efthimiou J, Johnston C, Spiro SG, Turner-Warnick M. Pulmonary disease in Behçet's syndrome. Q J Med 1986;227:259-80.

20. Lacombe P, Frija G, Parlier H, Lang F, Hamza M, Hamza R, et al. Transcatheter embolization of multiple pulmonary artery aneurysms in Behçet's syndrome. Acta Radiol Diagn 1985;26:251-3.

21. Salamon F, Weinberger A, Nilli M. Massive hemoptysis complicating Behçet's syndrome: the importance of early pulmonary angiography and operation. Ann Thorac Surg 1998;45:566-7.

22. Vansteenkiste JF, Peene P, Verschakelen JA, van de Woestijine KP. Cyclosporin treatment in rapidly progressive pulmonary thromboembolic Behçet's disease. Thorax 1990;45:295-6.

23. Huong DLT, Wechsler B, Papo T, Piette J C, Bletry O, Vitous J M, et al. Arterial lesions in Behçet's disease. A study in 25 patients. Rheumatol 1995;22:2103-13.

24. Durieux P, Bletry O, Huchon G, Wechsler B, Godeau P. Multiple pulmonary arterial aneurysms in Behçet's disease and Hughes-Stovin syndrome. Am J Med 1981;71:736-41.

25. Kaklamani V, Vaipouolos G, Kaklamanis P. Semin Arthritis Rheum 1998;27:197-212.

26. Casselman F, Meyns B, Herygers P, Verougstraete L, Van Elst F, Daenen W. Pulmonary artery aneurysm: is surgery always indicated? Acta Cardiol 1997;5:431-6.

27. Casselman F, Deferm $H$, Peeters $P$, Vanermen $H$. Aneurysm of the left pulmonary artery: surgical allograft repair. Ann Thorac Surg 1995; 60:1423-5.

28. Bartter T, Irwin RS, Nash G. Aneurysms of the pulmonary arteries. Chest 1988;94:1065-75

29. Barros WGP, Morone J r N, Brito RR, Martinez JAB. Regressão de aneurisma pulmonar na doença de Behçet. J Pneumol 1997;23:20810.

30. Magalhães IF, Kuroki IR, Lopes AJ, Albuquerque EMN, Klumb EM, Goulart AE. Aneurismas das artérias pulmonares na doença de Behçet: regressão após tratamento imunossupressor. J Pneumol 1999; 25:176-80.

31. Lorenzi Filho G, Barbas CSV, Carvalho CRR, Capelozzi VD, Gonçalves CR, Saldiva PHN, et al. J Pneumol 1990;16:155-60. 